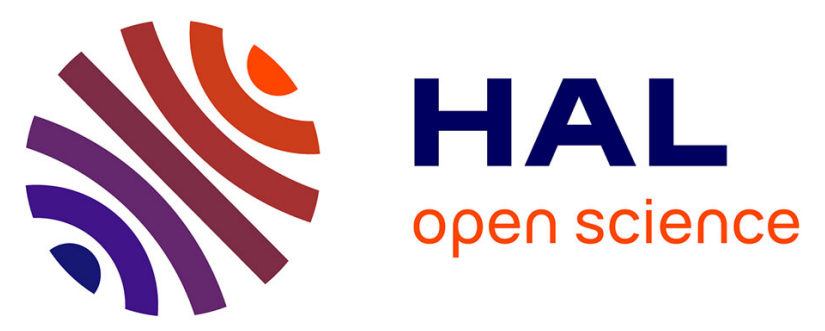

\title{
Serum keratin 19 (CYFRA21-1) links ductular reaction with portal hypertension and outcome of various advanced liver diseases
}

Karim Hamesch, Nurdan Guldiken, Mahmoud Aly, Norbert Hüser, Daniel Hartmann, Pierre Rufat, Marianne Ziol, Katharina Remih, Georg Lurje, Bernhard Scheiner, et al.

\section{To cite this version:}

Karim Hamesch, Nurdan Guldiken, Mahmoud Aly, Norbert Hüser, Daniel Hartmann, et al.. Serum keratin 19 (CYFRA21-1) links ductular reaction with portal hypertension and outcome of various advanced liver diseases. BMC Medicine, 2020, 18, pp.336. 10.1186/s12916-020-01784-7 . hal-03150962

\section{HAL Id: hal-03150962 \\ https://hal.sorbonne-universite.fr/hal-03150962}

Submitted on 24 Feb 2021

HAL is a multi-disciplinary open access archive for the deposit and dissemination of scientific research documents, whether they are published or not. The documents may come from teaching and research institutions in France or abroad, or from public or private research centers.
L'archive ouverte pluridisciplinaire HAL, est destinée au dépôt et à la diffusion de documents scientifiques de niveau recherche, publiés ou non, émanant des établissements d'enseignement et de recherche français ou étrangers, des laboratoires publics ou privés. 


\title{
Serum keratin 19 (CYFRA21-1) links ductular reaction with portal hypertension and outcome of various advanced liver diseases
}

Karim Hamesch ${ }^{1 \dagger}$, Nurdan Guldiken ${ }^{1 \dagger}$, Mahmoud Aly ${ }^{1,2}$, Norbert Hüser ${ }^{3}$, Daniel Hartmann ${ }^{3}$, Pierre Rufat ${ }^{4}$, Marianne Ziol ${ }^{5,6,7}$, Katharina Remih ${ }^{1}$, Georg Lurje ${ }^{8,9}$, Bernhard Scheiner ${ }^{10}$, Christian Trautwein ${ }^{1}$, Mattias Mandorfer ${ }^{10}$, Thomas Reiberger $^{10}$, Sebastian Mueller ${ }^{11}$, Tony Bruns ${ }^{1,12,13}$, Pierre Nahon ${ }^{14,15,16}$ and Pavel Strnad ${ }^{1 *}$

\begin{abstract}
Background: Keratins (Ks) represent tissue-specific proteins. K18 is produced in hepatocytes while K19, the most widely used ductular reaction (DR) marker, is found in cholangiocytes and hepatic progenitor cells. K18-based serum fragments are commonly used liver disease predictors, while K19-based serum fragments detected through CYFRA21-1 are established tumor but not liver disease markers yet. Since DR reflects the severity of the underlying liver disease, we systematically evaluated the usefulness of CYFRA21-1 in different liver disease severities and etiologies.

Methods: Hepatic expression of ductular keratins (K7/K19/K23) was analyzed in 57 patients with chronic liver disease (cohort i). Serum CYFRA21-1 levels were measured in 333 Austrians with advanced chronic liver disease (ACLD) of various etiologies undergoing hepatic venous pressure gradient (HVPG) measurement (cohort ii), 231 French patients with alcoholic cirrhosis (cohort iii), and 280 hospitalized Germans with decompensated cirrhosis of various etiologies (cohort iv).

Results: (i) Hepatic K19 levels were comparable among F0-F3 fibrosis stages, but increased in cirrhosis. Hepatic K19 mRNA strongly correlated with the levels of other DR-specific keratins. (ii) In ACLD, increased serum CYFRA21-1 associated with the presence of clinically significant portal hypertension (CSPH; HVPG $\geq 10$ $\mathrm{mmHg})(\mathrm{OR}=5.87$ [2.95-11.68]) and mortality ( $\mathrm{HR}=3.02$ [1.78-5.13]; median follow-up 22 months). (iii) In alcoholic cirrhosis, elevated serum CYFRA21-1 indicated increased risk of death/liver transplantation (HR=2.59 [1.64-4.09]) and of HCC (HR=1.74 [1.02-2.96]) over the long term (median follow-up 73 months). (iv) In decompensated cirrhosis, higher serum CYFRA21-1 predicted 90-day mortality (HR $=2.97$ [1.92-4.60]) with a moderate accuracy (AUROC 0.64), independently from established prognostic scores.

(Continued on next page)
\end{abstract}

\footnotetext{
* Correspondence: pstrnad@ukaachen.de

${ }^{\dagger}$ Karim Hamesch and Nurdan Guldiken contributed equally to this work.

${ }^{1}$ Medical Clinic III, Gastroenterology, Metabolic Diseases and Intensive Care, University Hospital RWTH Aachen, Pauwelsstr. 30, 52074 Aachen, Germany

Full list of author information is available at the end of the article
}

(c) The Author(s). 2020 Open Access This article is licensed under a Creative Commons Attribution 4.0 International License, which permits use, sharing, adaptation, distribution and reproduction in any medium or format, as long as you give appropriate credit to the original author(s) and the source, provide a link to the Creative Commons licence, and indicate if changes were made. The images or other third party material in this article are included in the article's Creative Commons licence, unless indicated otherwise in a credit line to the material. If material is not included in the article's Creative Commons licence and your intended use is not permitted by statutory regulation or exceeds the permitted use, you will need to obtain permission directly from the copyright holder. To view a copy of this licence, visit http://creativecommons.org/licenses/by/4.0/. The Creative Commons Public Domain Dedication waiver (http://creativecommons.org/publicdomain/zero/1.0/) applies to the data made available in this article, unless otherwise stated in a credit line to the data. 
(Continued from previous page)

Conclusions: Hepatic K19 mRNA and serum CYFRA21-1 levels rise in cirrhosis. Increased CYFRA21-1 levels associate with the presence of CSPH and reliably indicate mortality in the short and long term independently of conventional liver biochemistry markers or scoring systems. Hence, the widely available serum CYFRA21-1 constitutes a novel, DRrelated marker with prognostic implications in patients with different settings of advanced liver disease.

Keywords: Keratin, Biomarker, Chronic liver disease, Cirrhosis, Ductal reaction, Prognostic

\section{Background}

Keratins are the intermediate filaments of epithelial cells that are expressed in a tissue-specific manner and therefore constitute widely used biomarkers [1, 2]. Keratins are subdivided into type I proteins (including $\mathrm{K} 1-\mathrm{K} 8$ ) and type II proteins (including K9-K20), and both types assemble to form heteropolymers. As a consequence, each cell type has a characteristic type I-type II keratin repertoire [3]. For example, keratins 8/18 (K8/K18) are ubiquitously produced in single-layered epithelia while K7/K19 are found in some, but not all, simple epithelial cells $[1,2]$. In the liver, K19 constitutes the most widely used histologic marker of ductal/ductular reaction (DR) as it is expressed in cholangiocytes and hepatic progenitor cells (HPC) but not in adult hepatocytes $[1,4]$.

Upon tissue injury, keratins are released into the serum and therefore are well-known disease markers [5]. K18-based serum markers pose the most prominent keratin biomarkers as they represent useful surrogates of hepatocellular injury [5, 6]. Among them, M30/M65 are among the most widely used severity markers in multiple liver disorders [5]. In contrast, while serum CYFR A21-1 is widely available in routine laboratories as a tumor marker [7, 8], its usefulness in liver disease has not been systematically studied.

The liver possesses two fundamentally different strategies to recover from parenchymal losses, i.e., the division of mature hepatocytes and the progenitor cellbased DR $[9,10]$. Division of mature hepatocytes is typically seen after surgical liver resection, but may become impaired during chronic liver diseases, especially at stages with advanced liver scarring (fibrosis). In the latter, DR constitutes the predominant mode of parenchymal replenishment $[9,10]$. DR is driven by HPC that can differentiate to both biliary and hepatocellular lineage [11]. Since regeneration typically reflects the rate of parenchymal loss, the extent of DR mirrors the severity of the underlying liver disease $[9,10]$ and thus may predict the disease course $[10,12]$.

In line, multiple studies showed that the amount of K7/K19-positive cells correlates with the severity of liver disease and is of prognostic relevance [12-17]. However, these studies only evaluated the amount of DR-specific keratins in tissue sections, which are less commonly available due to the increasing use of non-invasive methods for diagnosing and staging liver fibrosis and portal hypertension. Hence, the aim of this study was to test whether a serum marker reflecting the extent of DR would be a valuable tool for prognostication. Thus, we systematically analyzed hepatic K19 expression in liver biopsies from various chronic liver diseases and assessed the significance of CYFRA21-1 serum levels in three further, well-characterized cohorts of patients with different settings of advanced liver disease.

\section{Methods \\ Analysis of human liver samples}

(Cohort i) 57 liver samples from patients with liver disease of different etiologies and 13 control samples were assessed for hepatic expression of the ductular keratins K19, K7, and K23 (Additional file 1: Table S1). Further details on patient selection and their molecular analysis are given in the supplement (Additional file 1).

\section{Analysis of CYFRA 21-1 levels in patients with different liver disease stages}

Three well-characterized cohorts were used to assess serum CYFRA21-1 in relation to liver disease severity and clinical outcomes: (cohort ii; Table 1) 333 Austrians with advanced chronic liver disease (ACLD; as defined by a hepatic venous pressure gradient (HVPG) $\geq 6 \mathrm{mmHg}$ [18]) of various etiologies undergoing HVPG measurement, (cohort iii; Table 2) 231 French patients with alcoholic cirrhosis, and (cohort iv; Table 3) 280 Germans hospitalized for decompensation of cirrhosis. Further details are given in the supplement (Additional file 1).

\section{Statistical analysis}

Continuous variables were given as median with interquartile range (IQR) and were compared by the Mann-Whitney $U$ test or Kruskal-Wallis test. Categorical variables were reported as absolute $(n)$ and relative (\%) frequencies, and contingency tables were analyzed with chi-squared tests.

The optimal cut-off values for CYFRA 21-1 as an indicator of $\mathrm{CSPH}$ or survival were determined using a cohort-specific Youden's index in receiver operating characteristic (ROC) analysis. Areas under the receiver operating characteristic curve (AUROC) were calculated. Univariate and multivariable analyses of risk factors for outcomes such as mortality were conducted using Cox 
regression analyses to calculate hazard ratios (HRs). Distributions among groups were assessed by univariable logistic regression analyses to calculate odds ratios (ORs), and forward-stepwise multiple logistic regression analyses were used to test for independent associations.

Time-to-event variables were displayed using KaplanMeier curves, and differences in event-free survival (or HCC occurrence, respectively) were tested using the logrank test. For the analysis of transplant-free survival, death or transplant was considered as endpoints and patients were right-censored at the end of the observation period.

HRs and ORs were given with their corresponding 95\% confidence intervals (CI) [in brackets].

Correlations between clinical variables and serum biomarkers or elastography parameters were assessed by calculating Spearman's rank correlation (Spearman's rho) coefficients.

Nominal $P$ values were reported for all statistical tests. Due to the exploratory nature of most parts of our study, we applied two-sided significance levels of $P<.05$ for all tests without correction for multiple testing. Statistical analyses were performed using the SAS System Package version 8.02 (SAS Institute, Cary, NC, USA) and SPSS version 23 (IBM, Armonk, NY, USA), and graphs were created with SPSS or Prism 5 (GraphPad, La Jolla, CA, USA).

\section{Results}

Hepatic K19 levels are upregulated in patients with liver cirrhosis

First, we analyzed the hepatic expression of ductular keratins $(K 7 / 19 / 23)$ in 57 patients with different liver fibrosis stages and disease etiologies (Additional file 1: Table S1). K19 mRNA levels were comparable among patients without fibrosis (F0) and with mild to advanced fibrosis stages (F1-F3), but elevated in cirrhotic individuals (F4; Fig. 1a). Notably, the increase seemed to be unrelated to the etiology of liver disease, since comparable K19 mRNA levels were seen in samples from patients with NAFLD, ALD, and chronic hepatitis C (Additional file 1: Fig. S1A). Similar results were obtained with the ductular keratins K7/K23 (Fig. 1b, c; Additional file 1: Fig. S1B, C). Moreover, the expression of $\mathrm{K} 7 / \mathrm{K} 19$ and K19/K23 strongly correlated with each other (Fig. 1d, e). The mRNA results were confirmed by immunohistochemistry and immunoblotting against K19 revealing a higher number of K19-positive cells and K19 protein levels in cirrhotic vs. control livers (Fig. If and Additional file 1: Fig. S2).

Taken together, levels of ductular keratins were elevated in patients with cirrhosis most likely as a consequence of a prominent DR.
Increased CYFRA21-1 associates with clinically significant portal hypertension (CSPH) and poor survival in patients with advanced chronic liver disease (ACLD)

To further assess the biological meaning and prognostic usefulness of serum CYFRA21-1 levels, we analyzed a thoroughly characterized Austrian cohort of 333 patients with ACLD of different etiologies. Two hundred eighty patients had CSPH (HVPG $\geq 10 \mathrm{mmHg}$ ), and these subjects had, as expected, higher Child-Pugh and model for end-stage liver disease (MELD) scores than their 53 counterparts without CSPH (Table 1). Serum CYFR A21-1 levels correlated with HVPG values in the overall cohort $(\mathrm{rho}=.375, P<.001)$. A similar correlation was seen in patients who experienced hepatic decompensation or liver-related death and those who did not (Fig. 2a). Accordingly, serum CYFRA21-1 was markedly higher in CSPH subjects (Table 1) and increased CYFR A21-1 values strongly associated with CSPH (CYFR A21-1 $\geq 3.90 \mathrm{ng} / \mathrm{mL}$ : unadjusted $\mathrm{OR}=5.87$ [2.95-11.68], $P<.001)$. Notably, the association between CYFRA21$1 \geq 3.90 \mathrm{ng} / \mathrm{mL}$ and $\mathrm{CSPH}$ remained highly significant after adjustment for etiology and demographic parameters (adjusted OR $[\mathrm{aOR}]=5.47$ [2.65-11.29], $P<.001$ ) Similarly robust associations were achieved after adjustment for platelets, as a simple non-invasive marker for portal hypertension, as well as the prognostic composite scores Child-Pugh and MELD (Table 4). An assessment of CYFR A21-1 as a dichotomized and continuous variable revealed comparable results (Table 2). Finally, CYFRA21-1 levels differed significantly between HVPG strata, i.e., ACLD patients without CSPH (i.e., HVPG 6-9 mmHg), HVPG $10-15 \mathrm{mmHg}$, and $\geq 16 \mathrm{mmHg}$ (Fig. 2b). The AUROC for serum CYFRA21-1 indicating CSPH was $0.75(0.69-0.82)$ while the AUROC for the composite score MELD was only slightly higher $(0.77(0.70-0.83))$. Combining CYFRA21-1 with MELD yielded a numerically higher AUROC (0.82 (0.76-0.88)) (Additional file 1: Suppl. figure S3).

In Kaplan-Meier analysis, CYFRA21-1 $\geq 3.90 \mathrm{ng} / \mathrm{mL}$ indicated a poor liver-related transplant-free survival over a median follow-up of 22 months (univariate Cox regression analysis: $\mathrm{HR}=3.02$ [1.78-5.13] $\log$-rank $P<.001$; Fig. 2c). Multivariable Cox regression analyses accounting for relevant confounders confirmed these associations (Additional file 1: Table S2). A similar association with liver-related death was observed when only patients with CSPH were considered (univariate $\mathrm{HR}=2.58$ [1.43-4.64], $\log$-rank $P=.002$; Fig. 2d).

Collectively, increased serum CYFRA21-1 levels were strongly and independently associated with the presence of CSPH and poor survival in patients with ACLD. Of note, CYFRA21-1 provided prognostic information in the subgroup of patients with $\mathrm{CSPH}$, highlighting its prognostic relevance beyond being a non-invasive surrogate of $\mathrm{CSPH}$. 

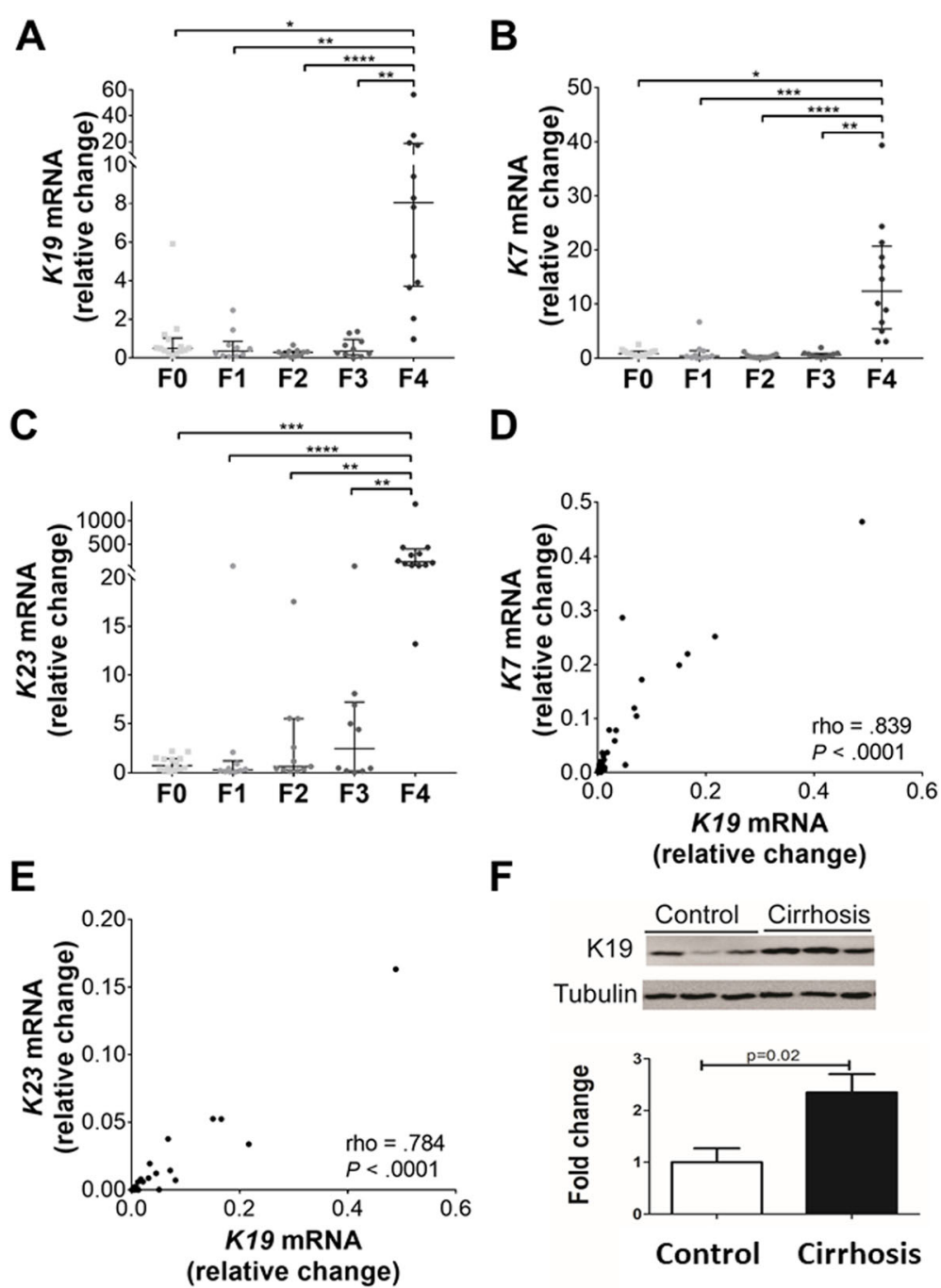

Fig. 1 Hepatic expression of genes related to ductular reaction in patients with chronic liver disease (cohort i). Fifty-seven livers from patients with various stages of liver fibrosis (fibrosis stages F0-F4) were analyzed (cohort i). a-c Relative hepatic expression of keratins 19, 7, and 23 (K19/ K7/K23), compared to RPLPO. Average K7/19/23 expression in control subjects was arbitrarily set as 1 and all other levels represent a ratio. d, e Spearman's correlation coefficient quantifies the relationship between the hepatic expression of K19 mRNA and K7 (d) or K23 (e). f K19 protein levels were determined by immunoblotting and tubulin was used as a loading control. The relative band intensity (lower panel) was quantified using ImageJ. ${ }^{*} P<.05,{ }^{* *} P<.01,{ }^{* *} P<.001,{ }^{* * *} P<.0001$

\section{CYFRA21-1 indicates poor long-term survival in alcoholic cirrhosis}

To validate the long-term prognostic usefulness of serum CYFRA21-1 levels, we turned to a French cohort of 231 patients with alcoholic cirrhosis, who were followed up longitudinally for HCC development (Table 3). During the median follow-up of 73 months, 98 patients died or received a liver transplant (non-survivors), while $133 \mathrm{pa}$ tients survived without the need for a liver transplant (transplant-free survivors; Table 3). While transplant-free survivors and non-survivors did not differ in their demographic characteristics (Table 3), non-survivors more often developed hepatocellular carcinoma ( $47.4 \%$ vs. $6.8 \%$, $P<.0001$; Table 3). $44.9 \%$ of non-survivors reached the pre-defined endpoint due to HCC while $51.0 \%$ did so due to other liver-related reasons. The baseline CYFRA21-1 serum level was higher in non-survivors vs. survivors (Table 3). A comparison of patients with high versus low serum CYFRA21-1 using the median $(5.26 \mathrm{ng} / \mathrm{mL})$ as a cut-off revealed that patients with higher CYFRA21-1 levels had more often ascites, had higher Child-Pugh scores, and reached the pre-defined endpoint of HCC more frequently (Additional file 1: Table S3).

In Kaplan-Meier analysis, CYFRA21-1 serum levels $\geq 5.26 \mathrm{ng} / \mathrm{mL}$ were associated with reduced cumulative overall transplantation-free survival (univariate $\mathrm{HR}=$ 2.59 [1.64-4.09], log-rank $P<.001$; Fig. 3a). Similar results were seen when only liver-related, not HCC- 
Table 1 Characteristics of the patient cohort with portal hypertension due to advanced chronic liver disease (ACLD), stratified by the presence of clinically significant portal hypertension (CSPH) (cohort ii)

\begin{tabular}{|c|c|c|c|c|}
\hline & $\operatorname{ACLD}(n=333)$ & No CSPH $(n=53)$ & $\operatorname{CSPH}(n=280)$ & $P$ value \\
\hline Age (years) & $54(15)$ & $55(14)$ & $54(15)$ & 0.51 \\
\hline Male sex $(n, \%)$ & $243(73)$ & $40(76)$ & $203(73)$ & .66 \\
\hline Alcohol $(n, \%)$ & $119(36)$ & $6(11)$ & $113(40)$ & $<.001$ \\
\hline Non-alcoholic fatty liver ( $n, \%)$ & $28(8)$ & $5(9)$ & $23(8)$ & \\
\hline Viral $(n, \%)$ & $141(42)$ & $38(72)$ & $103(37)$ & \\
\hline Child-Pugh score (points) & $6(3)$ & $5(0)$ & $7(4)$ & $<.001$ \\
\hline MELD score (points) & $10(5)$ & $8(2)$ & $11(5)$ & $<.001$ \\
\hline $\mathrm{ALT}(\mathrm{U} / \mathrm{L})$ & $41(51)$ & $61(66)$ & $40(46)$ & .001 \\
\hline AST $(U / L)$ & $59(53)$ & $60(52)$ & $58(53)$ & .97 \\
\hline Bilirubin (mg/dL) & $1.1(1.1)$ & $0.6(0.4)$ & $1.2(1.3)$ & $<.001$ \\
\hline Albumin (g/L) & $36.7(8.3)$ & $40.5(4.2)$ & $35.7(7.9)$ & $<.001$ \\
\hline INR & $1.20(0.30)$ & $1.10(0.18)$ & $1.30(0.22)$ & $<.001$ \\
\hline Creatinine (mg/dL) & $0.77(0.24)$ & $0.87(0.27)$ & $0.74(0.23)$ & .001 \\
\hline Platelet count (G/L) & $105(79)$ & $152(63)$ & $99(62)$ & $<.001$ \\
\hline HVPG $(m m H g)$ & $17(10)$ & $8(2)$ & $18(7)$ & $<.001$ \\
\hline CYFRA21-1 (ng/mL) & $4.3(3.3)$ & $3.0(1.5)$ & $4.6(3.3)$ & $<.001$ \\
\hline Liver-related death $(n, \%)$ & $70(21)$ & $10(19)$ & $60(21)$ & .68 \\
\hline Hepatic decompensation or liver-related death (n, \%) & $176(53)$ & $19(36)$ & $157(56)$ & .007 \\
\hline
\end{tabular}

Portal hypertension was defined as patients having a hepatic venous pressure gradient (HVPG) $\geq 6 \mathrm{mmHg}$ whereas CSPH was defined as HVPG $\geq 10 \mathrm{mmHg}$. Quantitative measures are shown as median (interquartile range) or as an absolute count ( $n$ ) and relative frequency (\%). Abbreviations: MELD model of end-stage liver disease, ALT alanine aminotransferase, AST aspartate aminotransferase, INR international normalized ratio

related endpoints were considered (univariate $\mathrm{HR}=$ 2.52 [1.65-3.85], $P<.001$; Fig. 3b). Using Cox's proportional hazards model, higher serum CYFRA21-1 levels (HR per $\mathrm{ng} / \mathrm{mL}$ increase 1.22 [1.02-1.43], $P=$ .02; Additional file 1: Table S4) along with higher age and higher Child-Pugh score were independently associated with overall death/need for transplantation. To further estimate the diagnostic accuracy of serum CYFRA21-1 for indicating mortality, we performed a ROC analysis. The corresponding AUROC was 0.60 (0.53-0.68) (Additional file 1: Suppl. figure S4), while the AUROC for the Child-Pugh score-which also encompasses clinical variables-was 0.63 (0.56-0.71).

Finally, higher CYFRA21-1 serum levels were associated with a somewhat higher occurrence of HCC (univariate $H R=1.74$ [1.02-2.96], log-rank $P=.039$; Fig. 3c). The positive predictive value and negative predictive value of the median CYFRA21-1 levels of $5.26 \mathrm{ng} / \mathrm{mL}$ for HCC occurrence were $22 \%$ and $74 \%$, respectively. Using the same Cox's proportional hazards model as for mortality, higher serum CYFRA21-1 levels were not significantly associated with $\mathrm{HCC}$ occurrence (Additional file 1: Table S4).

Taken together, in patients with alcoholic cirrhosis, serum CYFRA21-1 was an independent indicator of overall and liver-related long-term mortality.
CYFRA21-1 indicated short-term survival in patients with decompensated cirrhosis and ascites

To determine the predictive ability of serum CYFRA211 levels for short-term mortality in decompensated cirrhosis, we analyzed a cohort of patients hospitalized due to hepatic decompensation with ascites (Table 4). Within 90 days of follow-up, 88 patients (31.4\%) either died or were transplanted. These "non-survivors" were significantly older, more commonly had HCC or spontaneous bacterial peritonitis, and displayed higher MELD, Child-Pugh, and acute-on-chronic liver failure (ACLF) scores than transplant-free survivors (Table 4). Non-survivors had higher median CYFRA21-1 serum levels than survivors (Table 4). In the entire cohort, CYFRA21-1 only weakly correlated with ALT, AST, and MELD, while it did not significantly correlate with markers of liver synthetic function (e.g., INR and albumin; Additional file 1: Table S5). The CYFRA21-1 serum values were $8.5 \pm 12.5 \mathrm{ng} / \mathrm{mL}$ in cirrhotic patients without ACLF and $8.2 \pm 9.0 \mathrm{ng} / \mathrm{mL}, 12.4 \pm 13.4 \mathrm{ng} / \mathrm{mL}$, and $19.1 \pm 14.8 \mathrm{ng} / \mathrm{mL}$ in patients with ACLF grades 1,2 , and 3 , respectively (Additional file 1: Figure S5).

To estimate the diagnostic accuracy of serum CYFR A21-1 for 90-day transplant-free survival, we performed a ROC analysis. The AUROC for CYFRA 21-1 alone was 0.64 with an optimal cut-off being $12.19 \mathrm{ng} / \mathrm{mL}$ (sensitivity 


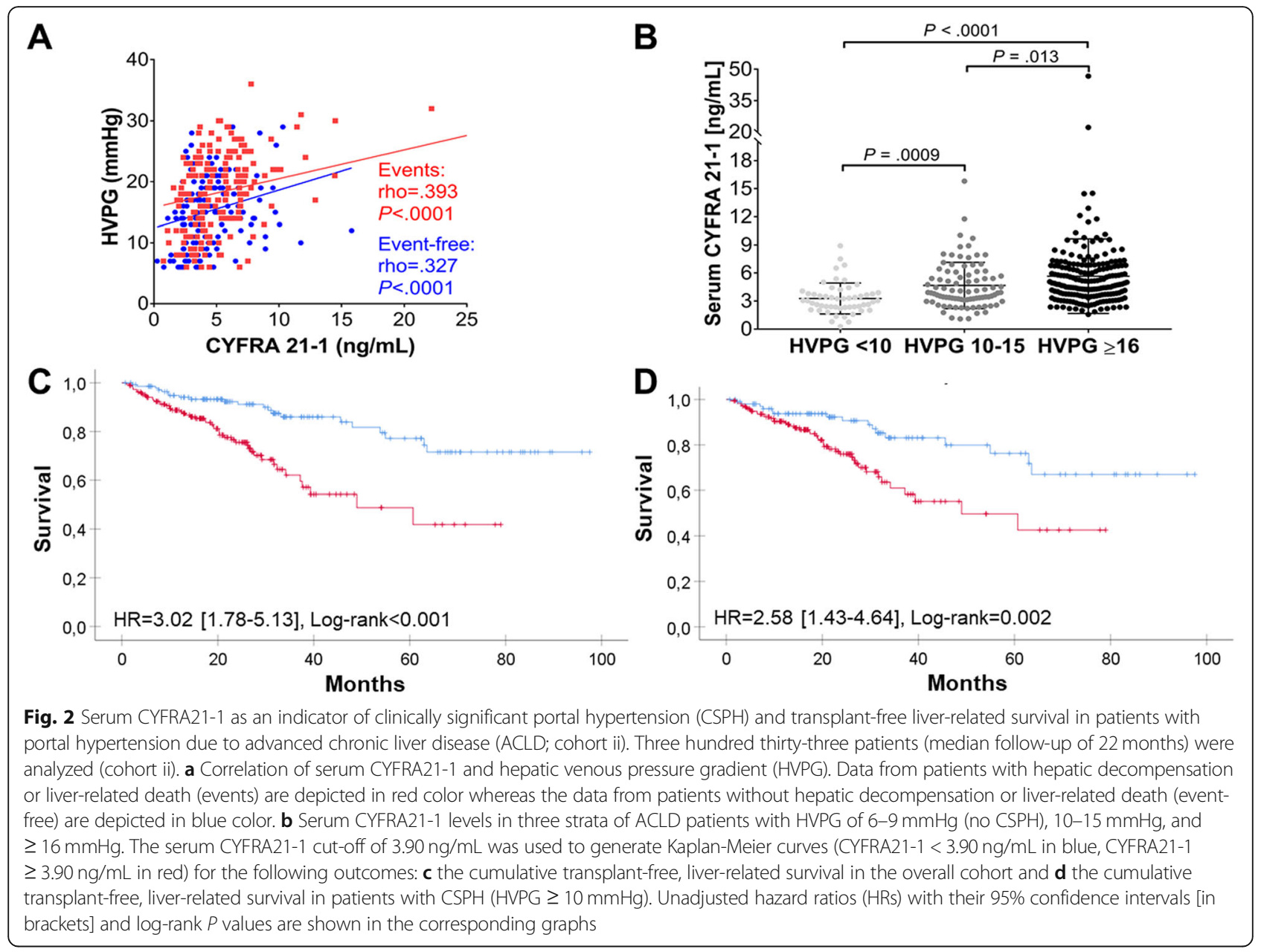

36.4\%; specificity 85.4\%; Additional file 1: Figure S6). High serum CYFRA21-1 at the established cut-off indicated patients with a low 90-day transplant-free survival in the corresponding Kaplan-Meier curve analysis (Fig. 4a). Notably, CYFRA21-1 levels in the highest quartile conferred a particularly bad prognosis (Fig. $4 \mathrm{~b}$ ). While the composite scores MELD (AUROC 0.70) and ACLF grade (AUROC 0.68) performed numerically better than CYFRA 21-1 alone, the combination of MELD with CYFRA 21-1 numerically improved the AUROC to 0.73 (Additional file 1: Figure S6).

In multivariable Cox regression models, high serum CYFRA21-1 (unadjusted HR 2.97 [1.92-4.60]; $P<.0001$ ) remained a robust indicator of death or transplant

Table 2 Binary logistic regression models for clinically significant portal hypertension (CSPH) using dichotomized and continuous variables for serum CYFRA21-1 in Patients with portal hypertension due to ACLD (cohort ii)

\begin{tabular}{|c|c|c|c|c|}
\hline & \multicolumn{2}{|c|}{$\begin{array}{l}\text { Serum CYFRA21-1, } \\
\text { dichotomized } \geq 3.90 \mathrm{ng} / \mathrm{mL}\end{array}$} & \multicolumn{2}{|l|}{$\begin{array}{l}\text { Serum CYFRA21-1, } \\
\text { per } n g / m L \text { increase }\end{array}$} \\
\hline & Odds ratio $(95 \% \mathrm{Cl})$ & $P$ value & Odds ratio $(95 \% \mathrm{Cl})$ & $P$ value \\
\hline Unadjusted & $5.87[2.95-11.68]$ & $<0.001$ & $1.73[1.40-2.14]$ & $<0.001$ \\
\hline Adjusted for age and sex & $6.19[3.09-12.39]$ & $<0.001$ & $1.77[1.42-2.20]$ & $<0.001$ \\
\hline Adjusted for etiology & $5.25[2.58-10.70]$ & $<0.001$ & $1.73[1.38-2.18]$ & $<0.001$ \\
\hline Adjusted for age, sex, and etiology & $5.47[2.65-11.29]$ & $<0.001$ & $1.79[1.41-2.28]$ & $<0.001$ \\
\hline Adjusted for platelet count & $6.74[3.19-14.22]$ & $<0.001$ & $1.75[1.40-2.18]$ & $<0.001$ \\
\hline Adjusted for MELD score & $4.07[1.99-8.33]$ & $<0.001$ & $1.53[1.23-1.90]$ & $<0.001$ \\
\hline
\end{tabular}

CSPH was defined as hepatic venous pressure gradient (HVPG) $\geq 10 \mathrm{mmHg}$. CYFRA21-1 was dichotomized using a cut-off determined by the maximum Youden index for the presence of CSPH. Abbreviations: CYFRA21-1 fragments of keratin 19, MELD model for end-stage liver disease 
Table 3 Characteristics of the patient cohort with cirrhosis due to long-term alcohol misuse included in HCC surveillance programs, stratified by liver-related survival (cohort iii)

\begin{tabular}{|c|c|c|c|c|}
\hline & Alcoholic cirrhosis $(n=231)$ & Transplant-free survivors $(n=133)$ & Non-survivors* $(n=98)$ & $P$ value \\
\hline Age at cirrhosis diagnosis (years) & $56.5(12.4)$ & $56.4(10.8)$ & $56.6(14.9)$ & .61 \\
\hline Male sex & $185(80.1 \%)$ & $105(78.9 \%)$ & $80(81.6 \%)$ & .64 \\
\hline BMI $\left(\mathrm{kg} / \mathrm{m}^{2}\right)$ & $27.0(6.0)$ & $27.0(5.9)$ & $27.5(7.0)$ & .07 \\
\hline Diabetes mellitus & $70(30.4 \%)$ & $38(28.6 \%)$ & $32(32.7 \%)$ & .50 \\
\hline Ascites & $87(37.4 \%)$ & $45(33.8 \%)$ & $42(42.9 \%)$ & .19 \\
\hline Hepatic encephalopathy & $23(10.0 \%)$ & $9(6.8 \%)$ & $14(14.3 \%)$ & .056 \\
\hline Child-Pugh score (points) & $6(3)$ & $6(3)$ & $7(3)$ & $<.001$ \\
\hline HCC during follow-up & $56(23.9 \%)$ & $9(6.8 \%)$ & $47(48.0 \%)$ & $<.001$ \\
\hline Survival (years) & $5.0(6.5)$ & $5.0(7.3)$ & $4.8(5.0)$ & .78 \\
\hline Liver transplantation & $17(7.4 \%)$ & - & $17(17.5 \%)$ & NA \\
\hline Liver-related death & $93(40.4 \%)$ & - & $93(95.9 \%)$ & NA \\
\hline Death & - & - & $98(100 \%)$ & NA \\
\hline HCC-related & & & 44 (44.9\%) & \\
\hline Liver-related & & & $50(51.0 \%)$ & \\
\hline Extra-hepatic & & & $4(4.1 \%)$ & \\
\hline $\operatorname{ALT}(\times \cup L N)$ & $1.0(1.0)$ & $1.0(1.0)$ & $1.0(1.0)$ & .28 \\
\hline AST $(\times$ ULN $)$ & $2.0(1.0)$ & $2.0(1.0)$ & $2.0(1.5)$ & .99 \\
\hline GGT $(\times$ ULN) & $4.0(6.0)$ & $4.0(6.0)$ & $5.0(6.0)$ & .45 \\
\hline Bilirubin $(\mu \mathrm{mol} / \mathrm{L})$ & $23.0(35.5)$ & $21.0(31.0)$ & $33.0(47.0)$ & .001 \\
\hline Albumin (mg/dL) & $36.3(10.3)$ & $39.0(9.4)$ & $34.0(10.1)$ & .001 \\
\hline PT (\% of control) & $65.0(30.5)$ & $68.0(27.0)$ & $57.0(30.5)$ & $<.001$ \\
\hline Platelet count (G/L) & $123.5(74)$ & $129.0(83.0)$ & $114.0(57.0)$ & .08 \\
\hline CYFRA21-1 (ng/mL) & $5.26(1.75)$ & $4.99(1.81)$ & $5.58(1.75)$ & .008 \\
\hline
\end{tabular}

Quantitative measures are shown as median (interquartile range) or as an absolute count ( $n$ ) and relative frequency (\%). Liver transaminases are displayed as a multiple of the upper limit of normal (ULN). Abbreviations: BMI body mass index, HCC hepatocellular carcinoma, ALT alanine aminotransferase, AST aspartate aminotransferase, GGT gamma-glutamyl transferase, $P T$ prothrombin time. *Non-survivors include patients who died or received a liver transplantation within the observation period (median follow-up of 73 months)

within 90 days after adjustment for MELD, MELD and age, ACLF grade and age, and MELD and white blood cell count (WBC) (Additional file 1: Table S6). Similar results were obtained when CYFRA21-1 was assessed as a dichotomized and a continuous variable (Additional file 1: Table S6). Sensitivity analyses showed that the prognostic ability of serum CYFRA21-1 remained strong across several patient subgroups such as patients with/without ACLF, high/low MELD score, and with/without HCC (Fig. 4c).

Collectively, in patients with decompensated cirrhosis, serum CYFRA21-1 constituted an independent indicator of 90-day transplant-free survival (Table 4).

\section{Discussion}

In our study, we evaluated the diagnostic relevance and prognostic significance of the K19-based CYFRA21-1 as a novel, direct serum marker of DR in different liver disease settings. We demonstrate that hepatic K19 expression remains unaltered in non-cirrhotic liver disease patients of various etiologies, which is in line with our previous findings [19]. In contrast, the expression of hepatocellular keratins K8/K18 rises already in intermediate fibrosis stages [19]. We also observed a strong correlation between the hepatic levels of K19 and the other DR keratins (K7/K23), thereby strongly suggesting that the detected elevated hepatic K19 expression mirrors an increased DR. Notably, DR is known to be particularly prominent in cirrhosis where regeneration through mitosis of mature hepatocytes becomes impaired $[9,10]$. Future studies correlating the hepatic expression of K19 with the serum levels of CYFRA21-1 in a well-characterized cohort of patients with various stages of chronic liver disease (i.e., non-advanced and advanced) are warranted.

The major aim of our study was to evaluate the pathophysiologic associations underlying the prognostic implications of CYFRA21-1 levels. Notably, CYFRA21-1 displayed only weak correlations with the hepatocellular injury markers AST/ALT or M30/M65 and no/minimal 
Table 4 Characteristics of hospitalized patients with decompensated cirrhosis and ascites, stratified by liver-related survival (cohort iv)

\begin{tabular}{|c|c|c|c|c|}
\hline & Decompensated cirrhosis $(n=280)$ & $\begin{array}{l}\text { Transplant-free survivors* } \\
\text { at } 90 \text { days }(n=192)\end{array}$ & $\begin{array}{l}\text { Non-survivors** } \\
\text { at } 90 \text { days }(n=88)\end{array}$ & $P$ value \\
\hline Age (years) & $59(52-67)$ & $57(48-64)$ & $63(55-69)$ & $<0.001$ \\
\hline Male sex & $206(74)$ & $142(74)$ & $64(73)$ & 0.88 \\
\hline Alcoholic liver disease & $220(79)$ & $158(82)$ & $62(70)$ & 0.03 \\
\hline $\begin{array}{l}\text { Self-reported alcohol use within the } \\
30 \text { days before admission }\end{array}$ & $128(46)$ & $91(47)$ & $37(42)$ & 0.44 \\
\hline $\mathrm{HCC}$ & $39(14)$ & $21(11)$ & $18(20)$ & 0.04 \\
\hline SBP & $37(13)$ & $14(7)$ & $23(26)$ & $<0.0001$ \\
\hline Child-Pugh C & $174(62)$ & $109(57)$ & $65(74)$ & $<0.01$ \\
\hline MELD score & $17(12-22)$ & $16(12-20)$ & $22(14-28)$ & $<0.000001$ \\
\hline ACLF & $73(26)$ & $34(18)$ & $39(44)$ & $<0.00001$ \\
\hline ACLF grade $(1 / 2 / 3)$ & $40(55) / 20(27) / 13(18)$ & $24(71) / 9(26) / 1(3)$ & $16(41) / 11(28) / 12(31)$ & $<0.01$ \\
\hline $\operatorname{ALT}\left(\mu \mathrm{mol} \mathrm{L}{ }^{-1} \mathrm{~s}^{-1}\right)$ & $0.56(0.38-0.88)$ & $0.56(0.38-0.77)$ & $0.58(0.41-1.16)$ & 0.07 \\
\hline AST $\left(\mu \mathrm{mol} \mathrm{L} \mathrm{L}^{-1} \mathrm{~s}^{-1}\right)$ & $1.07(0.70-1.86)$ & $1.04(0.68-1.71)$ & $1.12(0.73-2.55)$ & 0.14 \\
\hline Bilirubin $\left(\mu \mathrm{mol} \mathrm{L}^{-1}\right)$ & $40(20-97)$ & $37(23-87)$ & $78(24-173)$ & $<0.0001$ \\
\hline Albumin $\left(g \mathrm{~L}^{-1}\right)$ & $25(20-29)$ & $24(20-29)$ & $25(20-29)$ & 0.90 \\
\hline INR & $1.4(1.2-1.7)$ & $1.4(1.2-1.7)$ & $1.5(1.3-1.9)$ & $<0.001$ \\
\hline Creatinine $\left(\mu \mathrm{mol} \mathrm{L}{ }^{-1}\right)$ & $95(64-146)$ & $77(60-120)$ & $134(78-182)$ & $<0.000001$ \\
\hline Platelet count $\left(n L^{-1}\right)$ & $127(81-181)$ & $133(83-181)$ & $110(70-162)$ & 0.02 \\
\hline CYFRA21-1 (ng/mL) & $5.37(0.20-11.01)$ & $5.21(0.20-9.80)$ & $8.94(1.96-20.53)$ & $<0.0001$ \\
\hline
\end{tabular}

Quantitative measures are shown as median (interquartile range) or as an absolute count ( $n$ ) and relative frequency (\%). Abbreviations: HCC hepatocellular carcinoma, SBP spontaneous bacterial peritonitis, MELD model for end-stage liver disease, ACLF acute-on-chronic liver failure, $A L T$ alanine aminotransferase, AST aspartate aminotransferase, INR international normalized ratio. *Including 38 patients who were lost to follow-up after a median of 14 days. **Non-survivors include patients who died $(n=78)$ or received a liver transplantation $(n=10)$ within 90 days after study recruitment

correlation with liver synthesis parameters (Additional file 1: Table S5) suggesting that CYFRA21-1 mirrors liver disease severity rather than the synthetic ability of cirrhotic livers. This is in line with the current understanding of DR, that is thought to parallel liver disease severity $[10,11]$ as well as the studies employing immunohistochemical staining for ductular keratins, that also detected a correlation with liver disease severity $[12,17]$.

The extent of portal hypertension is an excellent indicator of liver disease severity and therefore has wellestablished prognostic implications [18]. Consequently, the predictive usefulness of CYFRA21-1 might be in part due to the fact that it correlates with the degree of portal hypertension. A correlation between DR and the extent of portal hypertension has been noted previously in a small series of patients with schistosomiasis (i.e., a cause of non-cirrhotic portal hypertension) undergoing direct portal pressure gradient [20] and is not surprising, since $\mathrm{DR}$ is known as the prevailing mode of regeneration in advanced/end-stage liver disease $[10,11]$. However, portal hypertension due to schistosomiasis differs in several aspects from the etiologies of chronic liver diseases that were evaluated by HVPG measurement in our study.
Regarding prognostic implications, in all three cohorts of patients with ACLD (cohorts ii-iv), higher CYFRA21-1 values were associated with reduced survival. This association was independent of the underlying etiology, the disease severity (i.e., compensated or decompensated cirrhosis), and a variety of potentially relevant confounders (e.g., demographic parameters). Importantly, the associations also remained robustly significant after adjustment for the MELD score, Child-Pugh score, and ACLF grade, indicating its incremental benefit over classical scoring systems. In line with this clear and consistent association of serum CYFRA 21-1 with liver-related survival, multiple studies demonstrated the usefulness of histological DR quantification in the long-term prognosis of chronic liver disease. These typically relied on the immunohistochemical detection of ductular keratins $\mathrm{K} 7 / \mathrm{K} 19$, as an indicator of the disease course [12-15]. Our findings extend these observations and demonstrate that DR products are useful not only as histological surrogates, but may also constitute attractive serum markers. This scenario is particularly attractive given that CYFRA21-1 is already widely available in the clinical routine, and thus, our findings are easy to implement. 


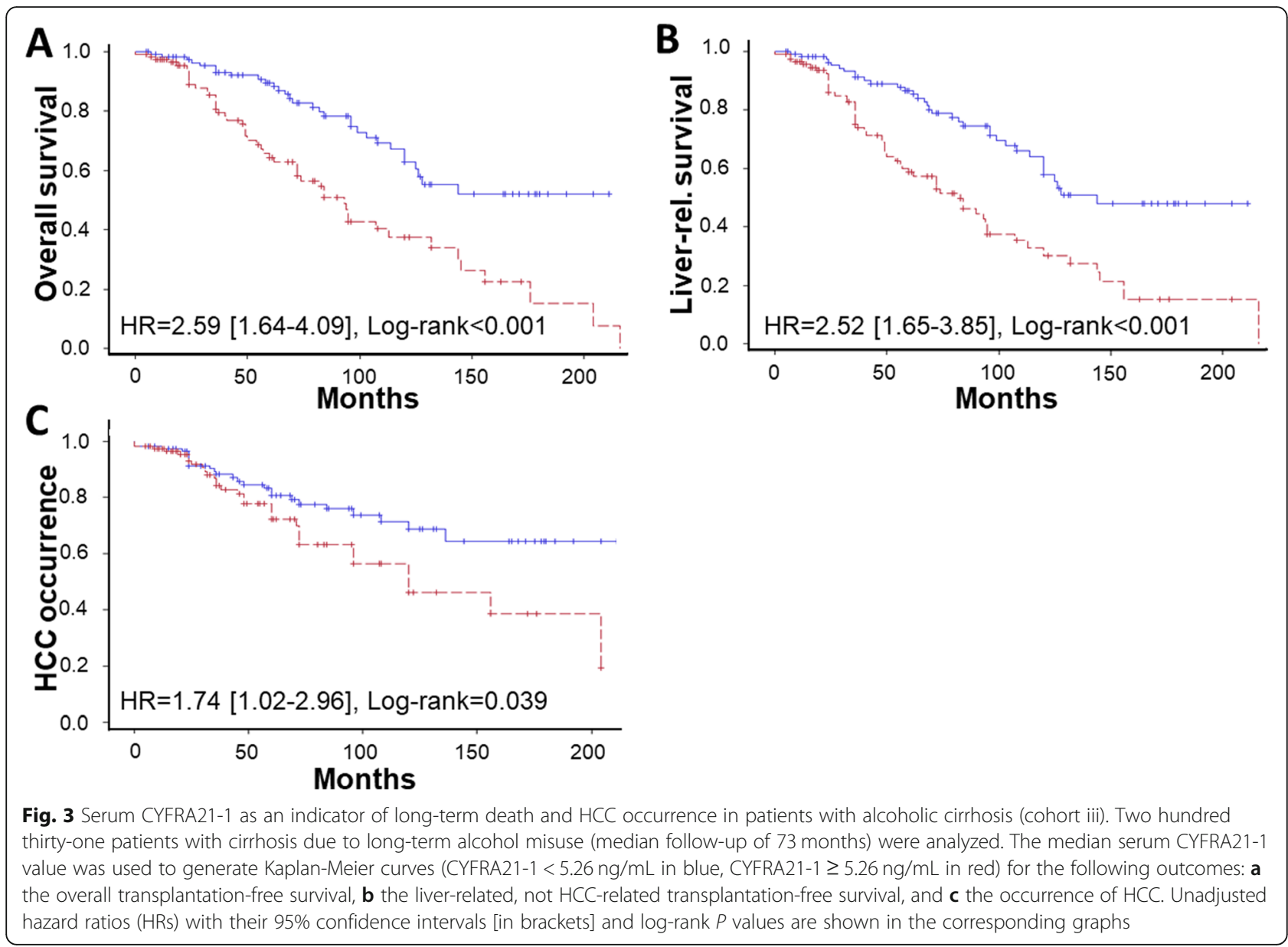

Notably, elevated CYFRA21-1 levels also associated with both HCC occurrence and HCC-unrelated liver-related mortality. While the association with HCC occurrence was rather weak, K19 is abundantly produced in liver cancer stem cells and delineates a HCC subgroup with poor prognosis [21-23].

In all three cohorts of patients with ACLD, the median CYFRA21-1 serum levels were mostly above the upper limit of normal for the general population (e.g., median CYFRA21-1 levels in healthy controls were described as of $1.5 \mathrm{ng} / \mathrm{mL}$ [24]). While $\mathrm{K} 19$ is produced in several non-hepatic tissues $[1,25]$, these data suggest that the release of K19 from cirrhotic livers exceeds the K19 liberation from other organs. However, our study design cannot fully exclude the contribution of non-hepatic sources of K19.

CYFRA21-1 does not appear to have a broad diagnostic range as a majority of values were below $6 \mathrm{ng} / \mathrm{mL}$. While continuous increases of serum CYFRA21-1 were significantly and independently associated with survival, the corresponding ORs and AUROCs were rather low (Additional file 1: Table 2, S4, and S6). Of note, using a cut-off determined by a cohort-specific Youden index (between 3.90 and $12.19 \mathrm{ng} / \mathrm{mL}$ ), CYFRA21-1's association with portal hypertension and survival was not only independent but also clear (i.e., ORs between 2 and 7). Given the steady hepatic K19 expression in noncirrhotic fibrosis stages (cohort i) and the increasing relevance of DR as a primary source of regeneration with increasing parenchymal loss and the concomitant tissue remodeling [9, 10], CYFRA21-1 does not appear to be discriminative in non-advanced chronic liver disease. Instead, CYFRA21-1's diagnostic utility lies rather in its association with $\mathrm{CSPH}$ and its independent survival prognostication when the serum levels are rather high. One might speculate that CYFRA21-1 is of particular prognostic relevance in decompensated cirrhosis and/or ACLF with a strong DR. However, further studies are warranted to determine CYFRA21-1's utility in prospective cohorts of patients with advanced and non-advanced chronic liver disease.

\section{Conclusions}

While CYFRA21-1 is a well-established and widely available serum marker of several non-hepatic tumors [5], our 


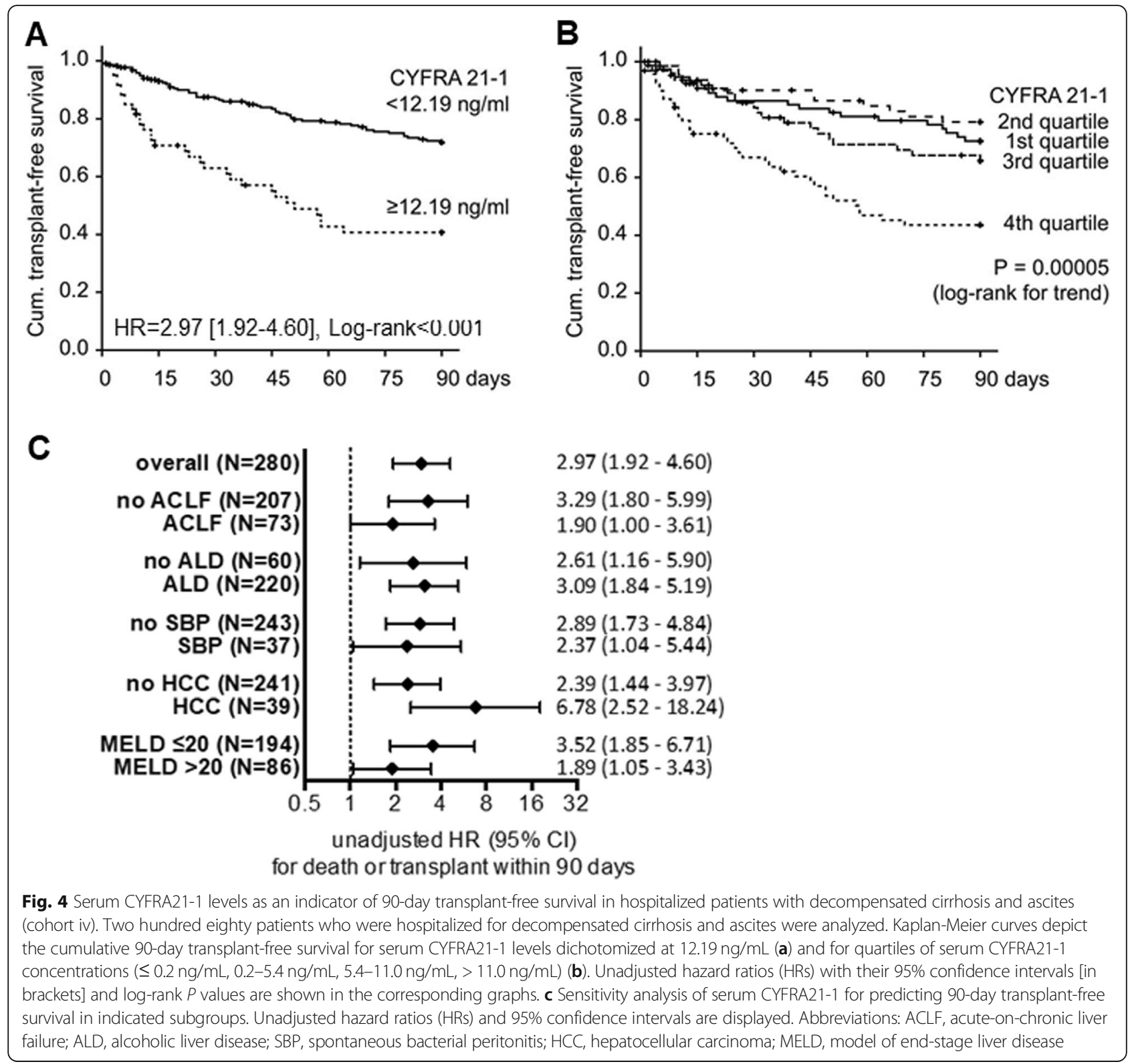

data strongly suggest that it can be repurposed to serve as the first direct serological marker of DR. Regardless of the underlying etiology, serum CYFR A21-1 levels are consistently elevated in different ACLD cohorts and are linked to the severity of portal hypertension. Furthermore, serum CYFRA21-1 levels appear to be a stable marker independent of conventional liver biochemistry markers and prognostic scores (e.g., MELD) pointing to an added benefit in terms of prognostication and pathophysiological insight. Importantly, CYFRA21-1 does not only reliably predict both the short- and long-term mortality of ACLD but also holds a great promise to promote our understanding of the biological importance of DR.

\section{Supplementary information}

Supplementary information accompanies this paper at https://doi.org/10. 1186/s12916-020-01784-7.

Additional file 1. Supplementary methods. Supplementary tables. Supplementary figures. References (Supplement).

\section{Abbreviations}

ACLD: Advanced chronic liver disease; ACLF: Acute-on-chronic liver failure; CPS: Child-Pugh score; CSPH: Clinically significant portal hypertension; CYFR A21-1: Keratin 19 fragments/fragments of keratin 19; DR: Ductular reaction/ ductal reaction; HCC: Hepatocellular carcinoma; HVPG: Hepatic venous pressure gradient; K19: Keratin 19; MELD: Model of end-stage liver disease; WBC: White blood cell count

\section{Acknowledgements}

We thank all patients for their participation in our study as well as Ingrid Breuer for excellent technical assistance. 


\section{Authors' contributions}

Study concept and design: K.H. and P.S.; acquisition of data: K.H., N.G., M.A., N.H., D.H., P.R., M.Z., G.L., B.S., M.M., T.R., S.M., T.B., and P.N.; investigation and methodology: K.H., N.G., and P.S.; data curation and validation: K.H.; analysis and interpretation of data: K.H., P.R., T.B., P.N., and P.S.; drafting of the manuscript: K.H. and P.S.; critical revision of the manuscript for important intellectual content: all authors; figures and tables: K.H., N.G., and T.B.; statistical analysis: K.H., P.R., and T.B.; obtained funding: K.H., C.T., S.M., T.B., and P.S.; project administration and study supervision: K.H. and P.S.; all authors had full access to all of the data. All authors read and approved the final version of this manuscript. All authors can take responsibility for the integrity of the data and the accuracy of the data analysis.

\section{Funding}

This work was supported by the German Research Foundation grant STR 1095/4-2 (to P.S.), MU 1373/9-1 (to S.M.), and BR 4182/3-1 (to T.B.); the Interdisciplinary Center for Clinical Research (IZKF) within the Faculty of Medicine at the RWTH Aachen University (to P.S.); the Else Kröner Excellence Fellowship (to P.S.); the Deutsche Forschungsgemeinschaft (DFG) consortium SFB/TRR57 "Liver fibrosis" (to P.S. and C.T.); the German Liver Foundation (to K.H.); and the START program within the medical faculty at RWTH Aachen University (to K.H. and N.G.).

Further remarks: We attest that we did not use any copyright protected material in our manuscript. No writing assistance was provided. Open Access funding enabled and organized by Projekt DEAL.

\section{Availability of data and materials}

The datasets used and analyzed during the current study are available from the corresponding author on reasonable request.

\section{Ethics approval and consent to participate}

For all cohorts, ethics approval and informed consent to participate in the study is available (cohort i: institutional review board of Aachen University, cohort ii: Ethikkommission der Medizinischen Universität Wien, cohort iii: Comité d'Ethique d'Aulnay-Sous-Bois, cohort iv: Ethikkommission des Universitätsklinikums Jena). All patients provided written informed consent, if the requirement of informed consent was not waived.

\section{Consent for publication}

\section{Not applicable.}

\section{Competing interests}

All authors declare no support from any organization other than the below mentioned ones for the submitted work, no financial relationships with any organizations that might have an interest in the submitted work in the previous 3 years, and no other relationships or activities that could appear to have influenced the submitted work. Hence, all authors declare themselves to be independent from funders with respect to this manuscript.

\section{Author details}

${ }^{1}$ Medical Clinic III, Gastroenterology, Metabolic Diseases and Intensive Care, University Hospital RWTH Aachen, Pauwelsstr. 30, 52074 Aachen, Germany. ${ }^{2}$ Department of Medicine and Infectious Diseases, Faculty of Veterinary Medicine, University of Sadat City, Sadat City, Egypt. ${ }^{3}$ Department of Surgery, Technical University of Munich, School of Medicine, Klinikum rechts der Isar, 81675 Munich, Germany. ${ }^{4}$ AP-HP, Service d'Biostatistic Hopital Jean Verdier, Bondy, France. ${ }^{5}$ Unité de Formation et de Recherche Santé Médecine et Biologie Humaine, Université Paris 13, Communauté d'Universités et Etablissements Sorbonne Paris Cité, Paris, France. ${ }^{6}$ Centre de Recherche des Cordeliers, INSERM, Sorbonne Université, USPC, Université Paris Descartes, Université Paris Diderot, F-75006 Paris, France. ${ }^{7}$ Centre de ressources biologiques du groupe hospitalier Paris-Seine-Saint-Denis, BB0033-00027, Hôpitaux Universitaires Paris-Seine-Saint-Denis, Assistance Publique Hôpitaux de Paris, Bondy, France. ${ }^{8}$ Department of Surgery and Transplantation, University Hospital Aachen, Aachen, Germany. ${ }^{9}$ Department of Surgery, Campus Charité Mitte | Campus

Virchow-Klinikum-Charité-Universitätsmedizin Berlin, Berlin, Germany. ${ }^{10}$ Vienna Hepatic Hemodynamic Lab, Division of Gastroenterology und Hepatology, Department of Internal Medicine III, Medical University Vienna, Vienna, Austria. ${ }^{11}$ Salem Medical Center and Center for Alcohol Research, University of Heidelberg, Heidelberg, Germany. ${ }^{12}$ Department of Internal
Medicine IV, Gastroenterology, Hepatology and Infectious Diseases, Jena University Hospital, Jena, Germany. ${ }^{13}$ Integrated Research and Treatment Center, Center for Sepsis Control and Care (CSCC), Jena University Hospital, Jena, Germany. ${ }^{14}$ AP-HP, Service d'Hépatologie, Hopital Jean Verdier, Bondy, France. ${ }^{15}$ Université Paris 13, Sorbonne Paris Cité, "Equipe labellisée Ligue Contre le Cancer", F-93206 Saint-Denis, France. " Inserm, UMR-1162,

"Génomique fonctionnelle des tumeur solides", F-75000 Paris, France.

Received: 18 February 2020 Accepted: 16 September 2020

Published online: 12 November 2020

\section{References}

1. Toivola DM, Boor P, Alam C, Strnad P. Keratins in health and disease. Curr Opin Cell Biol. 2015;32:73-81.

2. Jacob JT, Coulombe PA, Kwan R, Omary MB. Types I and II keratin intermediate filaments. Cold Spring Harb Perspect Biol. 2018;10(4). a018275. https://pubmed.ncbi.nlm.nih.gov/29610398/.

3. Haines RL, Lane EB. Keratins and disease at a glance. J Cell Sci. 2012;125(Pt 17):3923-8.

4. Yi H, Yoon HN, Kim S, Ku NO. The role of keratins in the digestive system: lessons from transgenic mouse models. Histochem Cell Biol. 2018;150(4):351-9.

5. Ku NO, Strnad P, Bantel H, Omary MB. Keratins: biomarkers and modulators of apoptotic and necrotic cell death in the liver. Hepatology. 2016;64(3): 966-76.

6. Vilar-Gomez E, Chalasani N. Non-invasive assessment of non-alcoholic fatty liver disease: clinical prediction rules and blood-based biomarkers. J Hepatol. 2018;68(2):305-15.

7. Holdenrieder S, Wehnl B, Hettwer K, Simon K, Uhlig S, Dayyani F. Carcinoembryonic antigen and cytokeratin-19 fragments for assessment of therapy response in non-small cell lung cancer: a systematic review and meta-analysis. Br J Cancer. 2017;116(8):1037-45.

8. Guida F, Sun N, Bantis LE, Muller DC, Li P, Taguchi A, et al. Assessment of lung cancer risk on the basis of a biomarker panel of circulating proteins. JAMA oncology. 2018;4(10):e182078.

9. Gilgenkrantz H, Collin de l'Hortet A. Understanding liver regeneration: from mechanisms to regenerative medicine. Am J Pathol 2018;188(6):1316-1327.

10. Sato K, Meng F, Giang T, Glaser S, Alpini G. Mechanisms of cholangiocyte responses to injury. Biochim Biophys Acta. 2018;1864(4 Pt B):1262-9.

11. Williams MJ, Clouston AD, Forbes SJ. Links between hepatic fibrosis, ductular reaction, and progenitor cell expansion. Gastroenterology. 2014; 146(2):349-56

12. Wendum D, Layese R, Ganne-Carrie N, Bourcier V, Merabtene F, Cagnot C, et al. Influence of progenitor-derived regeneration markers on hepatitis $C$ virus-related cirrhosis outcome (ANRS CO12 CirVir cohort). Hepatology. 2018;68(4):1534-48.

13. Ziol M, Nault JC, Aout M, Barget N, Tepper M, Martin A, et al. Intermediate hepatobiliary cells predict an increased risk of hepatocarcinogenesis in patients with hepatitis C virus-related cirrhosis. Gastroenterology. 2010; 139(1):335-43.e2.

14. Meriden Z, Forde KA, Pasha TL, Hui JJ, Reddy KR, Furth EE, et al. Histologic predictors of fibrosis progression in liver allografts in patients with hepatitis C virus infection. Clin Gastroenterol Hepatol. 2010;8(3):289-96, 96.e1-8.

15. Sancho-Bru P, Altamirano J, Rodrigo-Torres D, Coll M, Millan C, Jose Lozano J, et al. Liver progenitor cell markers correlate with liver damage and predict short-term mortality in patients with alcoholic hepatitis. Hepatology. 2012;55(6):1931-41.

16. Khan FM, Komarla AR, Mendoza PG, Bodenheimer HC Jr, Theise ND. Keratin 19 demonstration of canal of Hering loss in primary biliary cirrhosis: "minimal change PBC"? Hepatology. 2013;57(2):700-7.

17. Machado MV, Michelotti GA, Pereira TA, Xie G, Premont R, Cortez-Pinto H, et al. Accumulation of duct cells with activated YAP parallels fibrosis progression in non-alcoholic fatty liver disease. J Hepatol. 2015;63(4):962-70.

18. de Franchis R. Expanding consensus in portal hypertension: report of the Baveno VI Consensus Workshop: stratifying risk and individualizing care for portal hypertension. J Hepatol. 2015;63(3):743-52.

19. Guldiken N, Usachov V, Levada K, Trautwein C, Ziol M, Nahon P, et al. Keratins 8 and 18 are type $\|$ acute-phase responsive genes overexpressed in human liver disease. Liver Int. 2015;35(4):1203-12.

20. Pereira TA, Syn WK, Machado MV, Vidigal PV, Resende V, Voieta I, et al. Schistosome-induced cholangiocyte proliferation and osteopontin secretion 
correlate with fibrosis and portal hypertension in human and murine schistosomiasis mansoni. Clin Sci (Lond). 2015;129(10):875-83.

21. Kim H, Choi GH, Na DC, Ahn EY, Kim Gl, Lee JE, et al. Human hepatocellular carcinomas with "Stemness"-related marker expression: keratin 19 expression and a poor prognosis. Hepatology. 2011;54(5):1707-17.

22. Govaere O, Komuta M, Berkers J, Spee B, Janssen C, de Luca F, et al. Keratin 19: a key role player in the invasion of human hepatocellular carcinomas. Gut. 2014;63(4):674-85.

23. Li XF, Chen C, Xiang DM, Qu L, Sun W, Lu XY, et al. Chronic inflammationelicited liver progenitor cell conversion to liver cancer stem cell with clinical significance. Hepatology. 2017;66(6):1934-51.

24. Jiang AG, Chen HL, Lu HY. The relationship between Glasgow Prognostic Score and serum tumor markers in patients with advanced non-small cell lung cancer. BMC Cancer. 2015;15:386.

25. Moll R, Divo M, Langbein L. The human keratins: biology and pathology. Histochem Cell Biol. 2008;129(6):705-33.

\section{Publisher's Note}

Springer Nature remains neutral with regard to jurisdictional claims in published maps and institutional affiliations.

Ready to submit your research? Choose BMC and benefit from:

- fast, convenient online submission

- thorough peer review by experienced researchers in your field

- rapid publication on acceptance

- support for research data, including large and complex data types

- gold Open Access which fosters wider collaboration and increased citations

- maximum visibility for your research: over $100 \mathrm{M}$ website views per year

At BMC, research is always in progress.

Learn more biomedcentral.com/submissions 